\title{
Second Law Optimization of a PCM Based Latent Heat Thermal Energy Storage System with Tree Shaped Fins
}

\author{
A. Sciacovelli*, E. Guelpa, V. Verda \\ Department of Energy - DENERG \\ Politecnico di Torino \\ Email: adriano.sciacovelli@polito.it
}

\begin{abstract}
The aim of this paper is to perform a thermodynamic optimization of a Y shaped fin design used to improve thermal performance of a latent heat thermal energy storage (LHTES) unit. The investigation is performed through a CFD model that takes into account the thermal behavior of the system. Temperature and phase fields are obtained to characterize the heat transfer phenomenon and to compute the entropy generation rate within the system. Global entropy generation and energy flux are adopted as objective functions to perform a shape optimization of the $\mathrm{Y}$ shaped fins with angles and branches lengths that can vary freely. The optimization results indicate that a higher energy transfer is achieved by a fin configuration with long secondary branches with an orientation angle of $30^{\circ}$. This design allows one to increase PCM solidification rate of about 30\%. Furthermore, Y-shaped fins allow to increase the exergy flux released by the PCM, thus Second-law efficiency is not affected although entropy generation increases. This work represents the first detailed thermodynamic optimization of a system involving an unsteady process. This aspect is particularly important since a clear tendency of many energy systems is toward transient operation, thus design optimization methods should evolve accordingly.
\end{abstract}

\section{Keywords: Entropy generation minimization; numerical modeling; phase change; exergy; bifurcation.}

\section{Introduction}

One of the key aspects related with energy saving is the imbalance between energy demand and energy supply. This aspect is particularly marked for energy systems such as thermal power generation systems, heating \& power systems, solar energy utilization and air conditioning systems. Energy storage systems play a relevant role when it is required to match energy availability and the need. Energy storage is classified in three groups: sensible, latent and thermo-chemical heat storage. Among them, latent heat thermal energy storage (LHTES) is particularly attractive because of high energy storage capacity and the possibility of energy storage at nearly constant temperature. For summary of LHTES applications the interested reader can consult the papers [1] and [2].

LHTES units use phase change materials (PCMs) which undergo melting or solidification when energy is exchanged between the heat transfer fluid (HTF) and the PCM. Several typologies of PCMs have been studied in the past three decades. Organic and inorganic phase change materials have been considered for various applications [2] Paraffinic and non-paraffinic PCMs have several advantages such as chemical stability, low cost and congruent melting. Inorganic PCMs are typically salt hydrates which allow to significantly increase the energy storage capacity because of their high latent heat in comparison to organic PCMs. However, inorganic PCMs are corrosive and present incongruent melting.

Beside the attractive features, PCMs usually present poor thermo-physical properties and low thermal conductivity. As a consequence, melting and solidification rates can be unacceptably low and LHTES systems effectiveness is significantly influenced. A wide range of techniques have been proposed to enhance thermal performance of PCMs. Among the others, the use of PCMgraphite composites [3-5], encapsulation [6], and dispersion of nanoparticles [7-9]. However, most of the investigations concerns the use of extended surfaces (fins) to improve the performance of shell-and-tube LHTES units [10-14], since this configuration is closer to real PCM heat exchanger applications.

Beside conventional fin configurations, other shapes that mimic dendritic and branched structures have become popular. $\mathrm{Y}$ and $\mathrm{T}$ shape elements have been adopted in several applications [15-17] and promise a more homogeneous distribution of transport processes. However, the geometrical parameters characterizing the tree-shaped architecture must be carefully chosen in order to obtain optimal results. Among the other methods, thermodynamic optimization has become popular in thermal engineering, since it is based on Second-law analysis and entropy generation [18]. Such a kind of analysis is particularly suitable for investigating LHTES systems, as the real use of thermal storage is to store exergy, i.e. useful energy. Moreover, Second-law analysis allows one to investigate thermodynamic irreversibilities which directly affect the performance of engineering systems. Second-law investigation permits to evaluate the entropy generation which quantifies the destruction of useful energy. Exergy analyses of LHTES units have been performed by several authors: Shabgard et al. [19] performed energy and exergy analysis of a latent heat storage tank for solar power generation. The results show that irreversibilities can be reduced by choosing carefully the melting temperature of the PCM in conjunction with HTF inlet temperature. Ezan et al. [20] performed energy and exergy analyses for the 
charging process of an ice-on-coil thermal energy storage. The authors numerically evaluated energy/exergy effectiveness which indicate that thermal and flow parameters of the heat transfer fluid play a key role in determining the performance of the system. However, the local entropy generation rate, i.e. the local exergy destruction was not investigated. Ereck and Dincer [21] performed an entropy generation analysis of a latent heat storage system during the melting process. The authors indicated that entropy generation is crucial for LHTES and it should be carefully taken into account to increase system performance. However, they did not performed any design improvements on the basis of entropy generation results.

Although several studies of LHTES units based on exergy analyses are available, the literature lacks Secondlaw analyses of finned LHTES systems, as indicated in [22]. In the authors' knowledge only Guelpa et al. [23] performed a design improvement of a finned LHTES unit on the basis of entropy generation analysis. In this context, this paper aims at performing a thermodynamic optimization of a Y shaped fin architecture used to improve thermal performance of a cylindrical LHTES unit. The analysis is performed by means of a CFD model that takes into account of the thermal behavior of the system. Temperature and phase distributions are obtained to characterize the heat transfer phenomenon and to compute entropy generation rate within the system. Global entropy generation and energy flux are then adopted as objective functions in order to perform a shape optimization of the $Y$ shaped fins with angles and branch length that can vary freely. In the authors' knowledge this work represents the first detailed thermodynamic optimization of a system involving an unsteady process. This aspect is particularly important since a clear tendency of many energy systems is toward transient operation, thus design optimization methods should evolve accordingly.

\section{Mathematical Model}

\subsection{Governing Equations}

Figure 1 depicts the system considered in this paper: it consists of a vertical shell-and-tube LHTES unit. Water flows through the inner pipe and it acts as heat transfer fluid (HTF). The phase change material is placed in the space between the HTF pipe and the external shell. The discharge process, i.e. the PCM solidification, is considered in this work. The phase change material is completely liquid at the beginning of the process and at a temperature higher than melting point $T_{m}$. At time $\mathrm{t}>0$ water at temperature $T_{g}$ below the PCM melting temperature flows through the pipe. Consequently, an heat transfer rate is established between the PCM and water, until solidification is completed. As illustrated in Figure 1, a longitudinally finned tube was considered in the analysis. Four Y-shaped fins were placed along the HTF pipe. The inner tube has a diameter of $16 \mathrm{~mm}$, while the external shell has a diameter of $100 \mathrm{~mm}$.

In the analysis, natural convection in the liquid phase of the PCM was ignored, since only the solidification process is considered in this paper. Such an assumption was confirmed in Refs. [24,25], since discharge process of LHTES units is dominated by heat conduction. Natural convection only exists at the beginning of the process. Consequently, the analysis reported in the following sections is focused only on a 2D transversal cross section of the LHTES unit, as illustrated in Figure 1.

The numerical simulations performed in this work consider the following energy equation:

$$
\frac{\partial}{\partial t}(\rho h)=\nabla \cdot(k \nabla T)
$$

where $\rho$ is the PCM density, $T$ is the temperature, $k$ is the thermal conductivity and $h$ is the enthalpy.

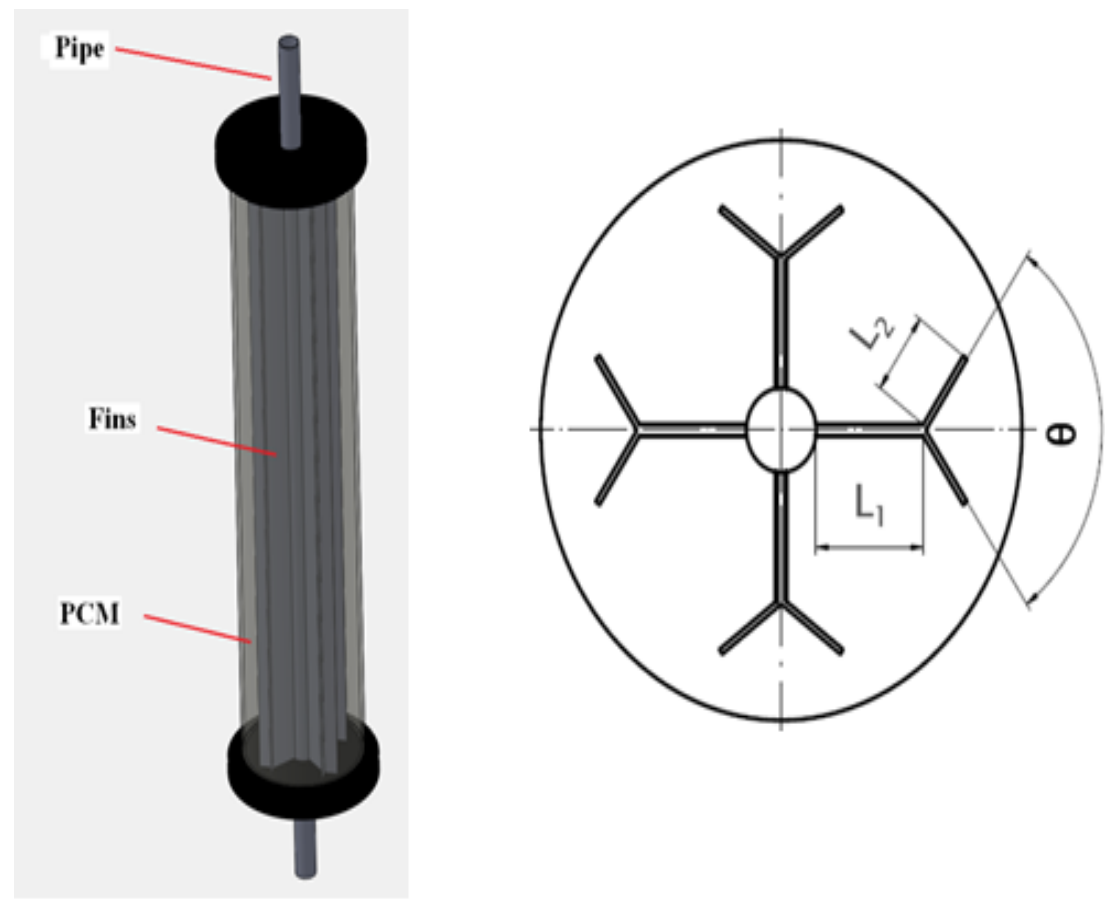

Figure 1. left) LHTES unit; right) fins cross section. 
The specific enthalpy of the PCM is expressed according to the enthalpy method [26,27]:

$$
h=h_{r e f}+\int_{T_{r e f}}^{T} c_{p} d T+\gamma L
$$

where $h_{\text {ref }}$ is the enthalpy at reference temperature $T_{\text {ref }}, L$ is the latent heat and $\gamma$ is the liquid fraction. The latter is defined as:

$$
\begin{array}{ccc}
\gamma=0 & \text { if } & T<T_{\text {solid }} \\
\gamma=1 & \text { if } & T>T_{\text {liquid }} \\
\gamma=\frac{T-T_{\text {solid }}}{T_{\text {liquid }}-T_{\text {solid }}} & \text { if } & T_{\text {solid }}<T<T_{\text {liquid }}
\end{array}
$$

Since the solidification process is dominated by heat conduction, the appropriated non-dimensional time $t^{*}$ is [28]:

$$
t^{*}=S t e \cdot \frac{\alpha \cdot t}{R^{2}}=S t e \cdot \text { Fo }
$$

where $\alpha$ is the thermal diffusivity and $R$ is the radius of the external shell. Stefan (Ste) and Fourier (Fo) numbers are defined as follows:

$$
\text { Ste }=\frac{c_{p} \cdot\left(T_{m}-T_{g}\right)}{L} \quad \text { Fo }=\frac{\alpha \cdot t}{R^{2}}
$$

The thermal behavior of the fins is described by means of the un-steady heat conduction equation. Aluminum fins were considered in the analysis $\left(k_{\text {fin }}=202 \mathrm{~W} / \mathrm{m} \mathrm{K}\right)$. The thickness of the main branch is $2 \mathrm{~mm}$. Secondary branches are $1 \mathrm{~mm}$ thick. A paraffin wax produced by SER S.p.A. was considered in the simulations. Its thermo-physical properties are reported in Table 1 . Variation of specific heat with temperature has been described by means of piecewise function.

Table 1. Thermophysical properties of the PCM.

\begin{tabular}{ll}
\hline Property & Value \\
\hline Melting temperature [K] & $323-325$ \\
Latent heat capacity [kJ/kg] & 250 \\
Thermal conductivity [W/mK] (solid-solid) & $0.15-0.15$ \\
Specific heat [kJ/kgK] & $1.8-2.3$ \\
Density (solid-solid) & $835-750$ \\
\hline
\end{tabular}

\subsection{Boundary Conditions and Numerical Approach}

Numerical modeling was conducted using the finite volume CFD code, FLUENT. Energy equation (1) is discretized by means of a second order central difference scheme. A second order implicit time integration scheme is used since it has the benefit of being unconditionally stable with respect of time step size. As indicated previously, the analysis here presented is focused only on a transversal cross section of the LHTES unit. Therefore, a 2D mesh was employed for the numerical simulations. Figure 2 presents the computational grid for a specific Y-shape fin configuration. An unstructured grid of 20k cells was found sufficient to ensure grid independent results. Furthermore, geometry presents a periodic structure. Thus it is possible characterize the system by considering just a sector of $90^{\circ}$. Adopted time step ranged between 0.1 and 0.2 since it was found sufficient to ensure convergence. Scaled residual of energy equation were monitored during the numerical computations and the solution was considered converged when the residual was lower than $10^{-8}$. Liquid PCM was considered at the initial temperature of $65^{\circ} \mathrm{C}$ and the LHTES unit was supposed to operate with a water inlet temperature of $20^{\circ} \mathrm{C}$. Along the boundary corresponding to the $\mathrm{HTF}$ pipe a temperature of $20^{\circ} \mathrm{C}$ was imposed. The outer shell was considered as adiabatic. Symmetry boundary conditions are imposed on the vertical and horizontal boundaries illustrated in Figure 2.

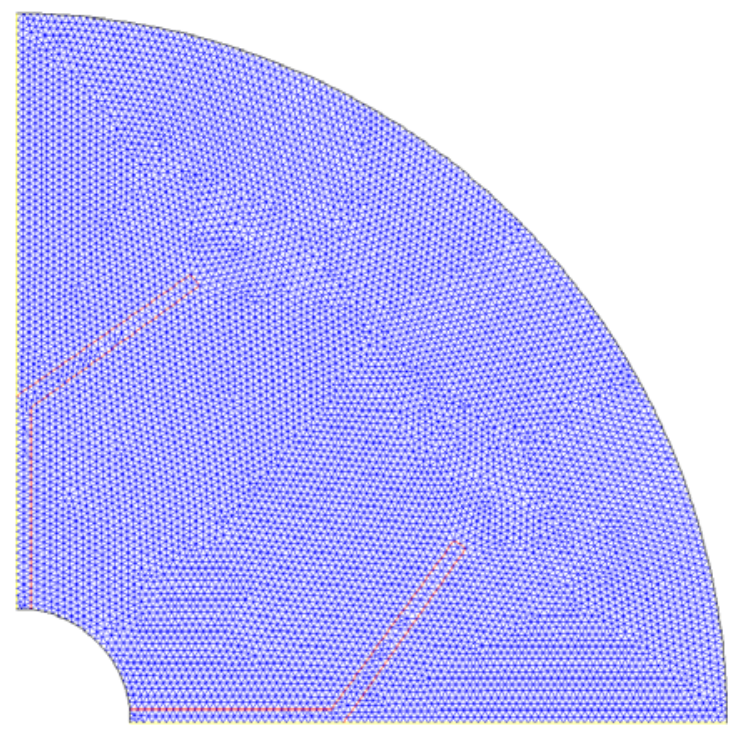

Figure 2. 2D computational grid.

\subsection{Entropy Generation and Shape Optimization}

The problem of optimal fins configuration inside a given system has been tackled by several researchers. Typical examples are heat exchangers, cooling devices and fuel cells [15,16,29,30]. Among the others, entropy generation is one of the possible indicators for selecting the optimal configuration of an energy system. The rate of entropy generation is the physical quantity that properly identifies the thermodynamic irreversibilities which cause system performance reduction. Entropy generation can be formulated in the context of non-equilibrium thermodynamics [31]. In particular, it is possible to write the entropy balance equation for an infinitesimal volume [31,32], that is:

$\rho \frac{D s}{D t}=-\nabla \cdot \vec{\sigma}+s_{p}$

where $s$ is the entropy per unit mass, $\vec{\sigma}$ is the entropy-flux vector and $s_{p}$ is the entropy generation rate per unit volume. In general, the entropy generation rate can be split into several contributions, each one related to a specific transport phenomenon. In the analysis here presented only heat transfer is considered, thus $s_{p}$ is formulated as:

$s_{p}=\frac{-\vec{J}_{q} \cdot \nabla T}{T^{2}}$ 
where $\vec{J}_{q}$ is the heat flux and it is formulated according to Fourier's law. After some algebraic manipulations it is also possible to show that:

$$
\vec{\sigma}=\frac{1}{T} \vec{J}_{q}
$$

The effect of the Y-shaped fins on heat transfer in the LHTES unit can be maximized by modifying the dimensions and orientation of the branches. Two factors influence the optimal fin design: the energy exchanged between PCM and fins and the thermodynamic irreversibilities associated to such heat transfer phenomenon. Since we are interested in the optimal configuration, it is necessary to parameterize the fin geometry and to define the design variables free to vary. Figure 1.right shows what was used: the lengths $L_{1}$ and $L_{2}$ of the two branches and the angle $\theta$ can vary. Fin configurations were considered symmetric along the direction of branch $L_{1}$. Furthermore, the total cross section area of Y-shaped fin was kept constant by adjusting the value of $L_{2}$. This reduces the number of free parameters to two, i.e. $L_{1}$ and $\theta$.

The optimization was performed considering two objective functions. The first one is the global entropy generation $S_{p}$, that is:

$$
S_{p}=\int_{0}^{\bar{t}} \int_{\Omega} s_{p} d \Omega d t^{*}
$$

The objective function $S_{p}$ is defined as a double integral of $s_{p}$ over the entire computational domain $\Omega$ and over the dimensionless time range $[0 \bar{t}] . S_{p}$ represents the entropy produced during solidification process over the time span corresponding to the dimensionless time range $[0 \bar{t}]$. The second objective function is:

$$
Q=\int_{0}^{\bar{t}} \int_{\Gamma} \vec{J}_{q} \cdot d \Gamma d t^{*}
$$

$Q$ is defined as the surface integral of heat flux $\vec{J}_{q}$ integrated over the dimensionless time range $[0 \bar{t}]$. The surface integral is extended over the boundaries corresponding to the fins surface and the pipe wall. $Q$ represents the amount of energy exchanged between the fins and the PCM over the time interval corresponding to the dimensionless time range $[0 \bar{t}]$. It can be noticed that both objective functions involve time integration. This is necessary since the solidification phenomenon that occurs in the LHTES unit is an unsteady process. Thus, in order to evaluate different fin configurations, it is necessary to take into account the effect of fin design on the time evolution of solidification. In the analysis this was done including time integration in the definition of the objective functions. Furthermore, $\bar{t}$ is chosen equal to 0.1 , which corresponds to $t=3000 \mathrm{~s}$. Therefore the time integration is performed only for a fraction of the entire process. This is necessary since the objective function $Q$ would be meaningless if the integration were performed for the entire solidification process. In fact the amount of energy stored in the system is the same for all the cases. It is desirable to maximize the amount of energy exchanged $Q$ and minimize the irreversibilities $S_{p}$. The optimization was performed by means of the Non-dominated Sorting Generic Algorithm version II (NSGA-II) [33]. Such genetic algorithm mimics some mechanism of biological evolution as reproduction, mutation, selection, etc. to find the optimal solution. The candidate solutions act as individuals in a population while objective function determines the fitness of each individual. Populations evolves through the repeated application of the above mechanism.

Finally, according to [21] it possible to define a second law efficiency $\psi$ starting from the previous two objective functions, namely

$\psi=1-N_{s}$

where $N_{s}$ is the entropy generation number and it is defined as:

$$
N_{s}=\frac{T_{0} S_{p}}{\int E x_{i n} d t^{*}}
$$

In the previous equation the denominator represents the exergy released by the PCM during solidification.

\section{Optimization Results}

Several geometries were analyzed during the optimization. Length $L_{1}$ was allowed to vary between $5 \mathrm{~mm}$ and $25 \mathrm{~mm}$ while angle $\theta$ varied between $0^{\circ}$ and $70^{\circ}$ during the optimization task. A scattered plot of global entropy generation $S_{p}$ versus heat $Q$ for the various cases is reported in Fig. 3. The plot confirms that a strong correlation exists between the two objective functions. It can be observed that a large amount of energy released by the PCM corresponds to a large entropy production and vice versa.

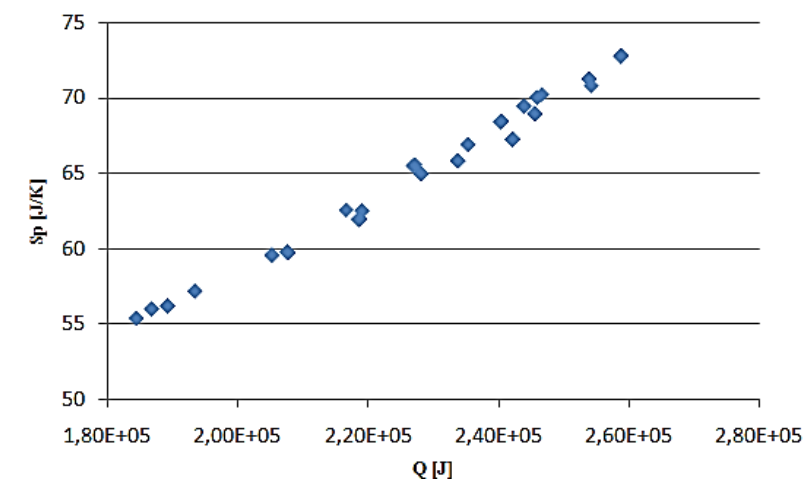

Figure 3. Entropy generation vs. energy exchanged.

The deviation between minimum and maximum values of $Q$ is about $0.8 \times 10^{5} \mathrm{~W}$, while $S_{p}$ ranges between 55 and 72 $\mathrm{W} / \mathrm{K}$. Such behavior is peculiar of the system here analyzed and a large entropy production is not necessarily a drawback. Thermodynamic performance of the system can be globally improved at constant heat flux by reducing the entropy generation. However, a second situation may occur: a design modification can bring an increase of entropy generation accompanied by a sufficient energy flux. This is not necessarily a disadvantage, if the increase in the thermodynamic irreversibilities is accompanied by a sufficient increase in the exergy flux.

In order to understand the influence of the design parameters on the objective functions a bubble chart is adopted. Such a plot is useful to show in a single figure data that have four dimensions. Figure 4 reports the values of 
design variables along the axes, the objective function $S_{p}$ is shown by the color map, while bubble diameter indicates objective function $Q$. A higher energy transfer is achieved by fin configurations with a small values of $L_{1}$. This means that a long secondary branch $\left(L_{2}\right)$ brings an improvement in the heat transfer between the PCM and the HTF. When the opposite situation occurs, i.e. large $L_{1}$ and small $L_{2}$, a lower entropy generation is achieved.

It can be observed from Figure 4 that the angle $\theta$ has some effect of the objective functions: if $L_{1}$ is larger than 9 $\mathrm{mm}$ an increase of $\theta$ brings an increase of both $S_{p}$ and $Q$. For small $L_{1}, S_{p}$ and $Q$ both augment if $\theta$ is increased from $10^{\circ}$ to $30^{\circ}$. However if $\theta>30^{\circ}$, a wider secondary branch angle brings a reduction of both objective functions.

Figure 5 shows the liquid fraction for two different fin designs investigated during the optimization. Fig. 5 (a) depicts the liquid fraction at $t^{*}=0.1$ for a fin design with a main branch of $25 \mathrm{~mm}$ and an angle $\theta$ of $70^{\circ}$. In this design, the secondary branch is very short since the fin cross section is kept constant for all the cases. It can be seen that the solidification front is aligned along the main branches, while most of the PCM is still liquid in the zone between the fins. In such a zone solidification is not particularly enhanced by fin design (a). This drawback can be mitigated if design (b) is adopted. Such a configuration has a short main branch and secondary branch of $25 \mathrm{~mm}$. Angle $\theta=$ $30^{\circ}$. In design (b) solidification rate is higher compared to design (a), since a long secondary branches properly orientated enhance solidification in the region between two fins.

(a)
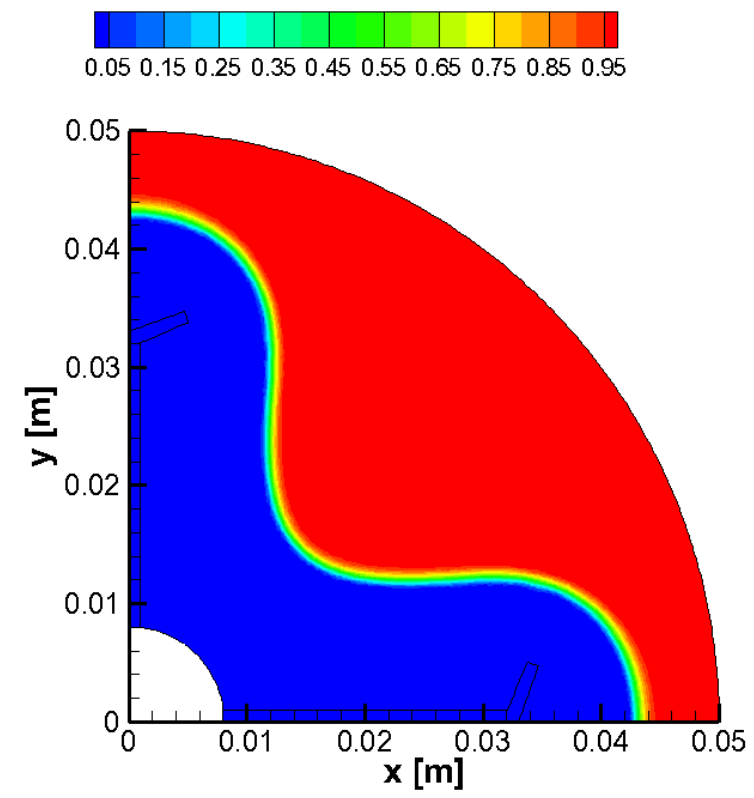

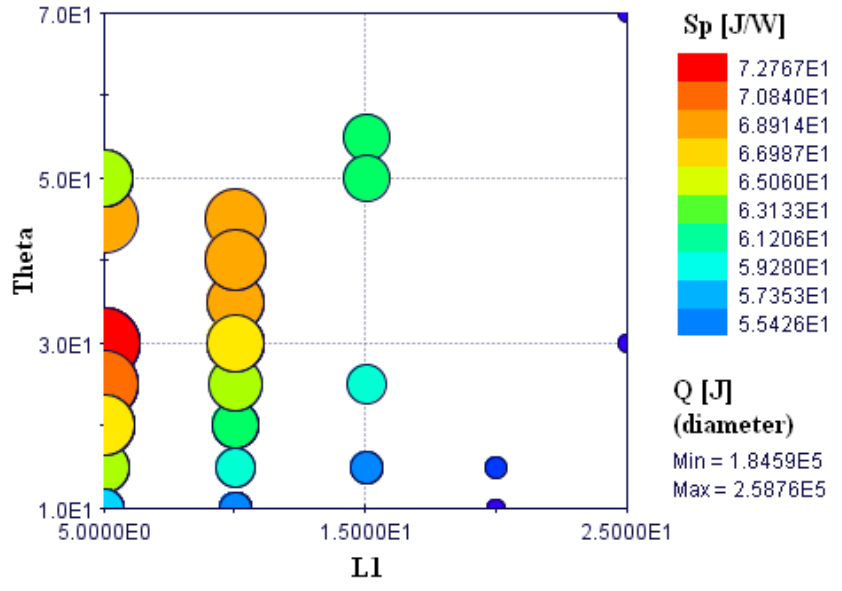

Figure 4. Bubble chart of entropy generation.

It can be also observed in Figure 5 that the solidification front position along the $x$ direction is not particularly affected by design (b) compared to (a). In fact solid PCM is found at about $x=42 \mathrm{~mm}$ for design (a) and at $x=38 \mathrm{~mm}$ for design (b). The latter significantly enhances PCM solidification in the entire LHTES: $41 \%$ of PCM is solid at $t^{*}=0.1$ when design (a) is adopted, while the amount of solid PCM increases to 59\% for design (b).

The temperature field at $t^{*}=0.1$ is shown in Figure 6 . From the contours it is possible to deduce that the average PCM temperature is lower for fin design (b) compared to design (a). This indicates that long secondary branches also improve sensible heat transfer rate between the PCM and the pipe wall. Thus, in a given time span, a larger amount of energy can be retrieved from PCM when fin design (b) is adopted.

(b) Lquid Fraction
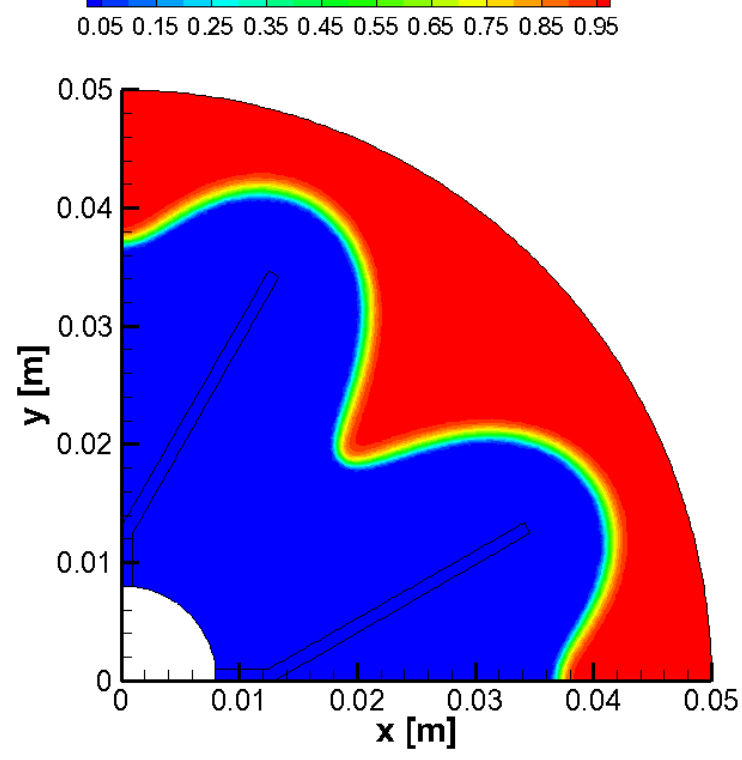

Figure. 5. (a) Liquid fraction at $t^{*}=0.1$ when $L_{1}=25 \mathrm{~mm}$ and $\theta=70^{\circ}$; (b) Liquid fraction at $t^{*}=0.1$ when $L_{1}=5 \mathrm{~mm}$ and $\theta=30^{\circ}$.

The designs illustrated in Figs. 5 and 6 can be also compared on the basis of the Second law analysis. According to the bubble chart of Fig. 4, design (a) is characterized by the lowest entropy generation, but also the smallest $Q$. Namely, the smallest amount of energy exchanged between PCM and fins. On the other hand, design (b) allows one to achieve a higher solidification rates and therefore an higher $Q$. However, global entropy generation $S_{p}$ is maximum among the cases considered during the optimization. Larger thermodynamic irrevesibilities are not necessarily a disadvantage. Secondlaw efficiency $\psi$ is about $45 \%$ for design (b) and $46 \%$ for 
design (a). The two cases have very similar efficiency, however heat transfer is significantly enhanced for design (b). This is due to the fact that both global entropy generation and heat transfer rate increase when fin configuration (b) is used. However, Second-law efficiency is unaffected since also the exergy flux increases.

Figure 7 illustrates the entropy generation rate at different time frames when $L_{1}=5 \mathrm{~mm}$ and $\theta=30^{\circ}$. In can be seen that maximum value of $s_{p}$ occurs at the beginning of the solidification process and entropy generation is significant only near the fins. This is due to the heat transfer rate established between the PCM and the fins. As a consequence, temperature gradients are found near fin walls. At $t^{*}=0.005$ thermodynamic irreversibilities are also relevant in the fins and in particular at the fin roots. As solidification proceeds $\left(t^{*}=0.05\right)$, local value of $s_{p}$ diminishes. However, entropy generation becomes relevant in a larger portion of the domain. In particular $s_{p}$ becomes relevant where the PCM is already solid. In fact, $s_{p}$ distribution assumes a shape similar to the solidification front. Finally, at $t^{*}=0.1$ entropy generation is negligible in most of the domain, except near fin tips where temperature gradients are still relevant, as can be observed in Figure 6 .
Figure 8 reports the liquid fraction distribution at different times when $L_{1}=5 \mathrm{~mm}$ and $\theta=30^{\circ}$. The plot allows one to understand time evolution of solidification front: it can be seen that at the beginning of the process solid PCM is found only near the fins and the pipe. Entropy generation is also relevant in the same zone, as depicted in Figure 6. As solidification progresses, the amount of solid PCM increases in the zone between the fins due to the presence of elongated fins. At late stage of the process, liquid PCM in found only in the outer region of the system, i.e. near the shell of the unit. At this instant of time, heat transfer is not particularity enhanced by the fins, since they are completely surrounded by solid PCM.

The temperature distribution at three instants of time is shown in Figure 9. It can be noticed that at $t^{*}=0.005$ a low temperature region is found near the fins. This significantly increases the solidification rate, since PCM phase change is not only initiated along the HTF pipe but also along the fins attached to the pipe. It is clear from Figure 8 that relevant temperature gradients are found were PCM is already solid. As solidification progresses, temperature distribution tends to became more homogeneous which also implies a reduction of local entropy generation, as shown in Figure 7.
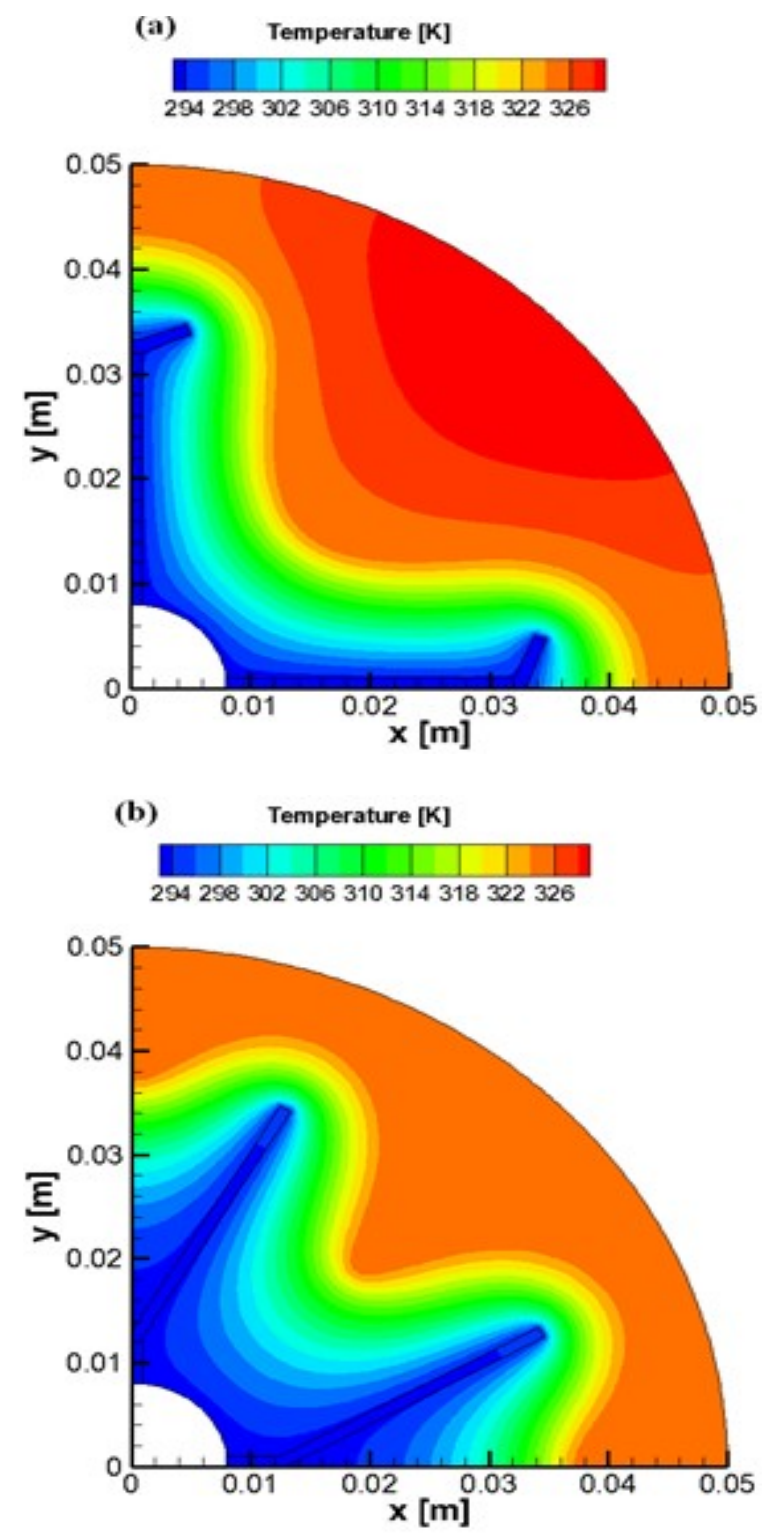

Figure. 6. (a) Temperature field at $t^{*}=0.1$ when $\mathrm{L} 1=25 \mathrm{~mm}$ and $\theta=70^{\circ}$; (b) temperature field at $t^{*}=0.1$ when $\mathrm{L} 1=5$ 

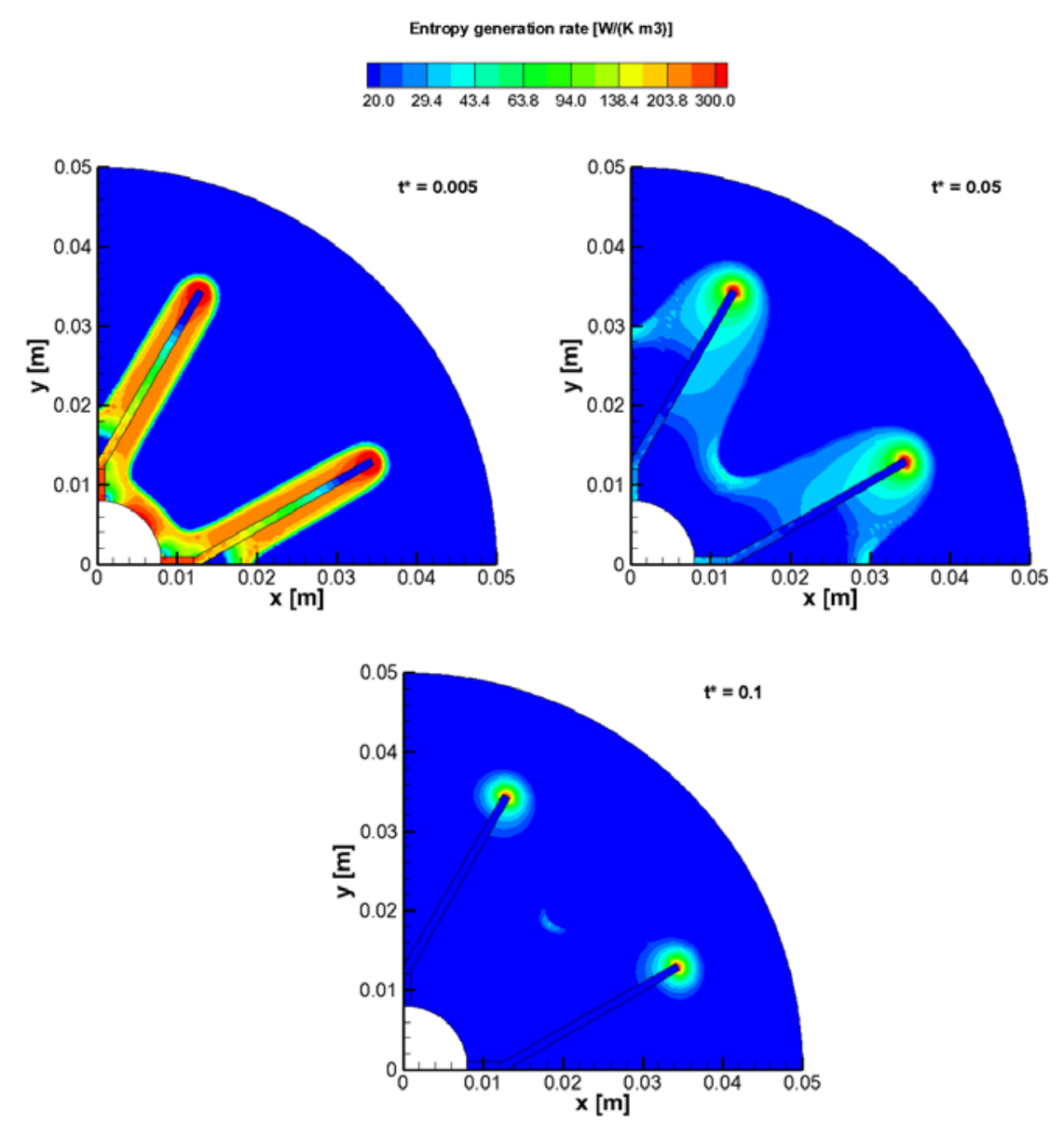

Figure 7. Entropy generation rate at different times; $L_{1}=5 \mathrm{~mm}$ and $\theta=30^{\circ}$.
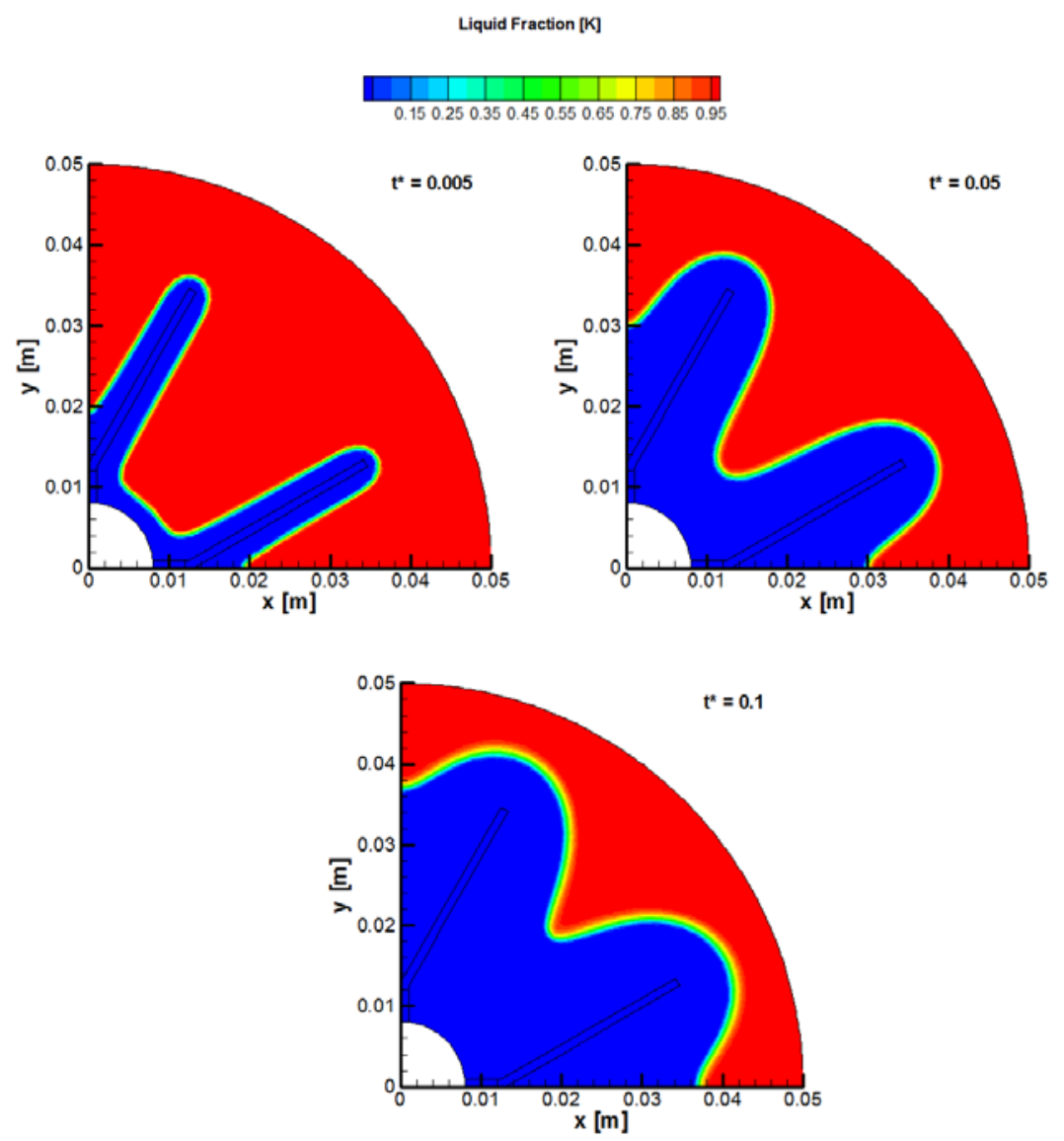

Figure 8. Liquid fraction at different times; $L_{1}=5 \mathrm{~mm}$ and $\theta=30^{\circ}$. 

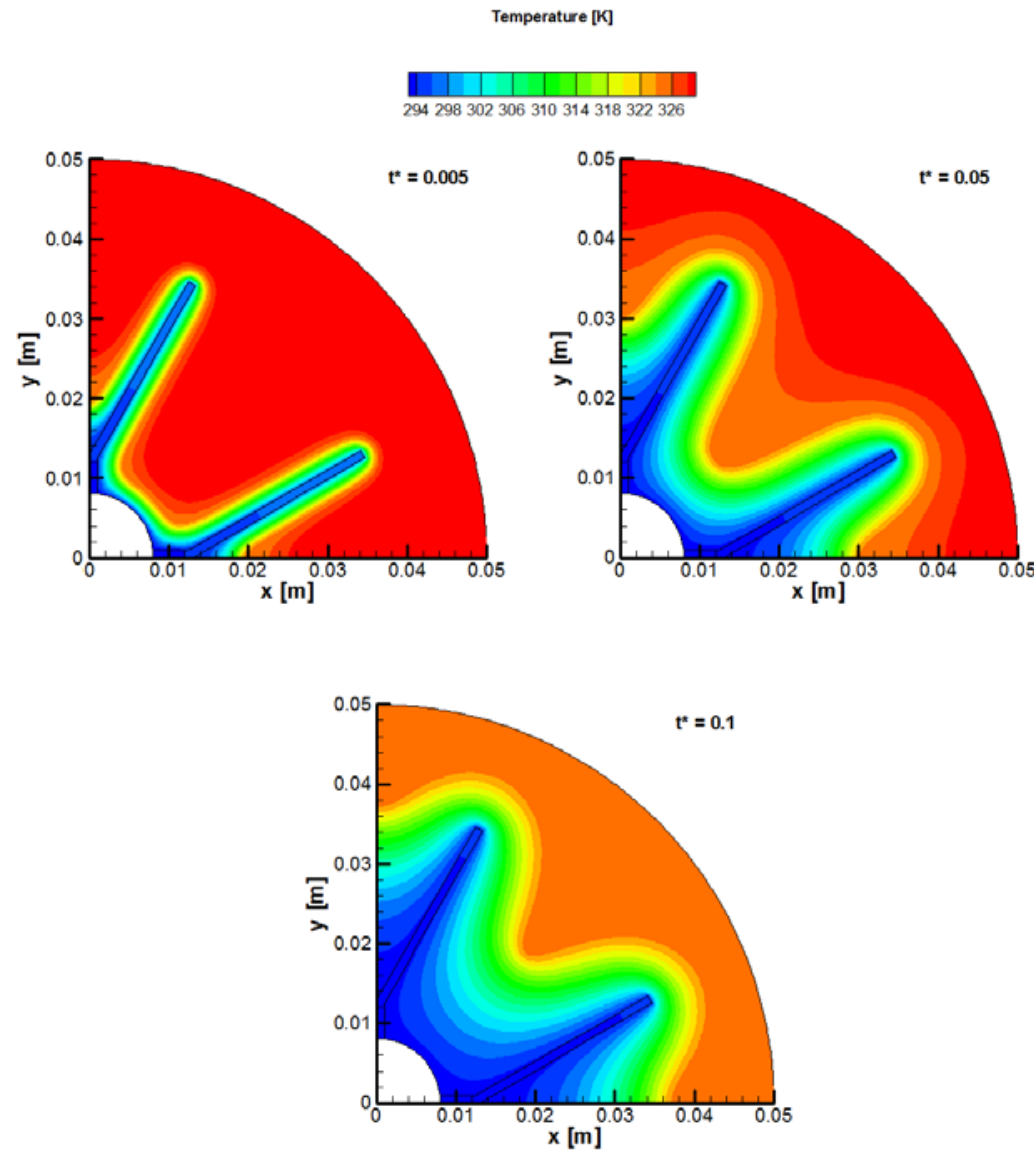

Figure 9. Temperature field at different times; $L 1=5 \mathrm{~mm}$ and $\theta=30^{\circ}$.

\section{Conclusions}

In this paper, entropy generation analysis is performed in order to optimize fins shape used to enhance PCM solidification in a latent heat storage system. The analysis was conducted by means of a 2D CFD model that takes into account the phase change problem through the enthalpy method. In order to enhance heat transfer in the system it is proposed to adopt Y-shaped fins. Fin shape optimization is performed using a genetic algorithm that takes into account two objective functions: the global entropy generation and the energy exchanged between the PCM and the fins in a given time interval.

The analysis shows that a strong correlation exists between the two objective functions: a large amount of energy released by the PCM corresponds to a large entropy production and vice versa. The optimization results indicate that higher energy transfer is achieved by fin configurations with long secondary branches and an orientation angle of $30^{\circ}$. This design allows one to increase the PCM solidification rate of about $30 \%$ with respect to radial fins. Such fin configuration is also characterized by relevant thermodynamic irreverisibilities, i.e. high entropy generation. However, this is not a disadvantage. In fact Second-law efficiency of the improved design is not reduced compared to radial fin design. This is due to the fact that $\mathrm{Y}$-shaped fins allows also to increase the exergy flux released by the PCM. Local entropy generation is also investigated in the paper. The results illustrate that the beginning of solidification process is characterized by high local values of entropy production. However, such thermodynamic irreversilities are found only near fin walls. As solidification proceeds, local entropy generation diminishes, but it becomes relevant in a larger portion of the system.

In conclusion, this paper shows that entropy generation analysis can be effectively used for the shape optimization of advanced thermal storage systems. This optimization must be performed in transient conditions, which is a novelty proposed here. This approach is shown to be effective even in the case of transient operation of energy systems. This is an important result, since there is a clear tendency in various modern energy systems towards transient operation.

\section{Nomenclature}

$\begin{array}{ll}c_{p} & \text { Specific heat }(\mathrm{J} / \mathrm{kg} \mathrm{K}) \\ F_{O} & \text { Fourier number } \\ h & \text { Specific enthalpy }(\mathrm{J} / \mathrm{kg}) \\ h_{\text {ref }} & \text { Reference specific enthalpy }(\mathrm{J} / \mathrm{kg}) \\ \vec{J}_{q} & \text { heat flux vector }\left(\mathrm{W} / \mathrm{m}^{2}\right) \\ k & \text { Thermal conductivity }(\mathrm{W} / \mathrm{m} \mathrm{K}) \\ L & \text { Specific latent heat }(\mathrm{J} / \mathrm{kg}) \\ N_{s} & \text { Entropy generation number } \\ R & \text { Radius (m) } \\ S_{p} & \text { Global entropy generation rate }(\mathrm{J} / \mathrm{K}) \\ S_{p} & \text { Local entropy generation rate }\left(\mathrm{W} / \mathrm{m}^{3} \mathrm{~K}\right) \\ S t e & \text { Stefan number } \\ T & \text { Temperature (K) } \\ T_{g} & \text { Inlet temperature }(\mathrm{K}) \\ T_{m} & \text { Melting temperature }(\mathrm{K}) \\ T_{\text {liquid }} & \text { Liquid Temperature }(\mathrm{K}) \\ T_{\text {solid }} & \text { Solid Temperature }(\mathrm{K}) \\ T_{0} & \text { Reference temperature }(\mathrm{K})\end{array}$


Time (s)

Dimensionless time

Greek symbols
$\alpha$
$\gamma$
$\rho$
$\vec{\sigma}$
$\psi$

Thermal diffusivity $\left(\mathrm{m}^{2} / \mathrm{s}\right)$

Liquid fraction

Density $\left(\mathrm{kg} / \mathrm{m}^{3}\right)$

Entropy flux vector $\left(\mathrm{W} / \mathrm{m}^{2} \mathrm{~K}\right)$

Second-law efficiency

\section{References}

[1] B. Zalba, J.M. Marin, L.F. Cabeza, H. Mehling, Review on thermal energy storage with phase change: materials, heat transfer analysis and applications, Appl. Thermal Eng., 23, 251-283, 2011.

[2] F. Agyenim, N. Hewintt, P. Eames, M. Smyth, A review of materials, heat transfer and phase change problem formulation for latent heat thermal energy storage systems (LHTESS), Renewable Sustainable Energy Reviews, 14, 615-628, 2010.

[3] L. F. Cabeza, H. Mehling, S. Hiebler, F. Ziegler, Heat transfer enhancement in water when used as PCM in thermal energy storage, Appl. Thermal Eng., 22, 1141-1151, 2002.

[4] S. Pincemin, R. Olives, X. Py, M. Christ, Highly conductive composites made of phase change materials and graphite for thermal storage, Solar Energy Mater. Solar Cells, 92, 603-613, 2008.

[5] F. Colella, A. Sciacovelli, V. Verda, Numerical analysis of a medium scale latent energy storage unit for district heating systems, Energy, 45, 397-406, 2012.

[6] A. F. Regin, S. C. Solanki, J. S. Saini, Heat transfer characteristics of thermal energy storage system using PCM capsules: A review, Renewable Sustainable Energy Reviews, 12, 2438-2458, 2008.

[7] E. S. Mettawee, G. M. R. Assassa, Thermal conductivity enhancement in a latent heat storage system, Solar Energy, 81, 839-845, 2007.

[8] S. Kalaiselvam, R. Parameshwaran, S. Harikrishnan, Analytical and experimental investigations of nanoparticles embedded phase change materials for cooling application in modern buildings, Renewable Energy, 39(1), 375-387, 2012.

[9] A. Sciacovelli, F. Colella, V. Verda, Melting of PCM in a thermal energy storage unit: numerical investigation and effect of nanoparticle enhancement, Int. J. Energy Res., 37, 1610-1623, 2012.

[10] L. Fan, J.M. Khodadadi, Thermal conductivity enhancement of phase change materials for thermal energy storage: A review, Renewable Sustainable Energy Reviews, 15, 24-46, 2011.
[11] M. Lacroix, Study of the heat transfer behavior of a latent heat thermal energy storage unit with a finned tube, International Journal of Heat Mass Transfer, 36, 2083-2092, 1993.

[12] A. Erek, Z. Ilken, M. A. Acar. Experimental and numerical investigation of thermal energy storage with a finned tube, International Journal of Energy Research, 29, 283-301, 2005.

[13] F. Agyenim, P. Eames, M. Smyth, A comparison of heat transfer enhancement in a medium temperature thermal energy storage heat exchanger using fin, Solar Energy, 83, 1509-1520, 2009.

[14] A. Al-Abidi, S. Mat, K. Sopian, M. Y. Sulaiman, Internal and external fin heat transfer enhancement technique for latent heat thermal energy storage in triplex tube heat exchangers, Applied Thermal Engineering, 53, 147-156, 2013.

[15] A. Bejan, S. Lorente, Constructal law of design and evolution: Physics, biology, technology, and society, Journal of Applied Physics, 113, 151301, 2013.

[16] A. Sciacovelli, C. Amelio, C. Repetto, V. Verda, G. Diaz, Performance Improvement of a circular MCFC through optimal design of the fluid distribution system, J. Fuel Cell Sci. Technol., 9, 041011, 2012.

[17] H. Kobayashi, S. Lorente, R. Anderson, A. Bejan, Trees and serpentines in a conductive body, Int. J. Heat Mass Transfer, 56, 4887-4494, 2013.

[18] A. Bejan, Entropy Generation Minimization. Boca Raton, FL: CRC Press, 1996.

[19] H. Shabgad, T. L. Bergman, A. Faghri, Exergy analysis of latent heat thermal energy storage for solar power generation accounting for constraints imposed by long-term operation and the solar day, Energy, 60, 474484, 2014.

[20] M.A. Ezan, A. Erek, I. Dincer, Energy and exergy analyses of an ice-on-coil thermal energy storage system, Energy, 36, 6375-6386, 2011.

[21] A. Erek, I. Dincer, An approach to entropy analysis of a latent heat storage module, Int. J. Thermal Sci., 47, 1077-1085, 2008.

[22] S. Jegadheeswaran, S. D. Pohekar, T. Kousksou, Exergy based performance evaluation of latent heat thermal storage system: A review, Renewable Sustainable Energy Reviews, 14, 2580-2595, 2010.

[23] E. Guelpa, A. Sciacovelli, V. Verda, Entropy generation analysis for the design improvement of a latent heat storage system, Energy, 53, 128-138, 2013.

[24] S. Jegadheeswaran, D. P. Sanjay, Performance enhancement in latent heat thermal storage system: a review, Renewable Sustainable Energy Reviews, 13, 2225-2244, 2009. 
[25] S. Kuravi, J. Trahan, M. M. Rahman, D. Y. Goswami, E. K. Stefanokos, Analysis of the transient heat transfer in a thermal energy storage module. in Proceedings of the ASME International Mechanical Engineering Congress \& Exposition 2010 IMECE10, Boston, USA, 1251-1258, 2010.

[26] V. R. Voller, C. Prakash, A fixed grid numerical modeling methodology for convection-diffusion mushy region phase-change problems, International Journal of Heat and Mass Transfer, 30, 1709-1719, 1987.

[27] A. Al-Abidi, S. B. Mat, K. Sopian, M. Y. Sulaiman, A.Th. Mohammed, CFD applications for latent heat thermal energy storage: a review, Renewable and Sustainable Energy Reviews, 20, 353-363, 2013

[28] B. J. Jones, D. Sun, S. Krishnan, S. V. Garimella, (2006). Experimental and numerical study of melting in a cylinder, International Journal of Heat and Mass Transfer, 49, 2724-2738, 2006.

[29] V. D. Zimparov, A. K. da Silva, A. Bejan, Thermodynamic Optimization of Tree-Shaped Flow Geometries, International Journal of Heat Mass Transfer, 49, 1619-1630, 2006.

[30] A. Sciacovelli, V. Verda, Entropy Generation Minimization for the Optimal Design of the Fluid Distribution System in a Circular MCFC, International Journal of Thermodynamics, 14(4), 167-177, 2011.

[31] S. R. de Groot, P. Mazur, Non-equilibrium thermodynamics. NY: Dover Publications, 2011.

[32] A. Sciacovelli, V. Verda, Entropy Generation Minimization in a Tubular Solid Oxide Fuel Cell, Journal of Energy Resources and Technology, Transaction of the ASME, 132(1), 0126011-01260111, 2010.

[33] D. Kalyanmoy, A Fast and Elitist Multiobjective Genetic Algorithm, IEEE Transaction on evolutionary computation, 6, 182-197, 2002. 